\title{
Wie Menschen mit Demenz und ihre Partner verbleibende Fähigkeiten nutzen können, um ihre Beziehung zu erhalten
}

Hlorian Bödecker (Ulm)

\section{Zusammenfassung}

Zicl des vorliegenden Artikels ist die Beantwortung der Frage, welche Fählhgkeiten bei Demenzkranken zur Aufrechterhaltung von intimen Beziehungen erhalten bleihen. D)azu werden zunächst durch cine Begriffsanalyse die wesentlichen Merkmale von intimen Beziehungen bestimnt. Befunde aus empirischen Studien zeigen anschließend, inwieweit diese Merkmale trotz dementieller Veränderungen bewahrt werden können. So bleibt die Fähigkeit, Emotionen zu crleben und auszudrücken, noch lange erhalten. Auch implizites emotionales Lernen und ein lange erhaltenes implizites (icdächtnis sind Ressourcen für die Aufrechterhaltung der Berichung zum partner.

Stichworte: 1)emenz, interpersonale Beriehungen, Pflegende, soziale Kompetenz, Bezichungsfähigkeit

Abstract: How people with dementia and their partners can use remaining abilities to maintain their relationship

The article focuses on an investigation into the emotional abilities which people with dementia are able to retain so that intimate relationships may be preserved. First, a conceptual analysis is carried out to define the essential characteristics of intimate relationships. Second, an overview of empirical results shows how intimate relationships can be maintained despite the changes brought about by dementia. In fact, people with dementia stay capable of experiencing and expressing cmotions for quite a long period. Equally, implicit emotional learning and the implicit memory are resources partners and the person with dementia can draw on to maintain their (emotional) relationship.

Key words: Dementia, Interpersonal relations, Caregivers, Social skills, Relational competence 


\section{Die Bedeutung der intimen Zweierbeziehung für Menschen mit Demenz}

Bezichungsforscher betonen, dass intime Beziehungen fundamental scien für unsere Lebenszufriedenheit und unser geistiges und körperliches Wohlbefinden. Gerade im Alter sei es wichtig, befriedigende Beziehungen zu anderen Menschen eingchen und erhalten zu können, weil man mit dem Schrumpfen sozialer Netzwerke konfrontiert sei, ein Bediurfnis nach sinnvoller, sozialer Beschäftigung habe und stärker von der Unterstiutzung anderer abhängig sei (vgl. Hansson et al. 2004). Insbesondere Menschen mit Demenz können von ihren Unterstützern einerseits ein Gefühl von Wert und Identität bekommen, andererseits jedoch auch bevormundet und durch Konfrontation mit Defixiten entwertet werden (vgl. Mackae 2011).

Für Menschen mit Demenz ist deshalb die Fähigkeit entscheidend, Bezichungen zu gestalten und aufrechterhalten zu kömen, die es ihnen erlauben, ihre Bedürfnisse nach Autonomie, lcbensstilkontinuität, Sicherheit und sozialer Akzeptanz zu befriedigen (vgl. Davics u. Nolan 2008; Stcchl 2006). Wenn Menschen mit Demenz einerseits stärker auf die Unterstïtzung für sie wichtiger Personen angewiesen sind, gleichzeitig aber durch multiple kognitive Einschränkungen sowie Veränderungen im Erleben und Verhalten becinträchtigt sind, stellt sich die lrage, welche Möglichkeiten für Menschen mit Demenz und ihre Partner bestehen, für alle Beteiligten befriedigende Bezichungen cinzugehen und zu erhalten.

Im Folgenden gebe ich deshalb einen Überblick darüber, wodurch intime Beziehungen charakterisiert sind und welche zugrundeliegenden Fähigkeiten vorhanden sein müssen, um ein Mindestmaß an Intinität erreichen zu können. Schließlich zeige ich auch, welche verbleibenden Fähigkeiten Menschen mit bemenz haben, die es ermöglichen, mit anderen intimo Beziehungen zu halten. Mittels der philosophischen Methode der Begriffsanalyse (vgl. Damschen u. Schönecker 2013), die versucht, die notwendigen Bestimmungen cines Begriffs herauszufinden, die zusammen hinreichend für scinen (iebrauch sind, untersuche ich zunächst, welche ligenschatten eine Beziehung mindestens haben muss, damit man von ciner intincn Zweierberiehung sprechen kann. Daraus leite ich dann die notwendigen Fähigkeiten beider Partner ab, die für cin Minimum an Intimität vorhanden sein müssen. Befunde aus empirischen Studien zeigen abschließsend, inwicweit diese lähigkeiten trotz der demenziellen Veränderungen erhalten bleiben können.

\section{Was macht intime Beziehungen aus?}

Bezichungsforscher unterscheiden zwischenmenschliche Bezichungen unter anderem danach, wo sic auf dem Kontinuum zwischen unpersönlichen Beziehungen auf der cinen Seite und intimen bzw. romantischen Bezichungen auf cler anderen Seite licgen. Inncrhalb dieses Spektrums werden meist die intimen Bezjehungen untersucht, weil sich hier beide partner besonders stark becinflussen und entsprechend bedeutsam für das eigene geistige und körperliche Wohlbefinden sind. In diesem Sinne definieren Bradbury und Karney intime Beriehungen wie folgt: "An intimate relationship is one characterized by strong, sustained, mutual influence across a wide range of interactions, featuring at least the potential for sexual interaction « (Bradbury u. Kanney 2010,11 ).

Dieser Definition lassen sich wesentliche Elemente von intimen Beziehungen entrichmen, die aber noch nicht hinreichend sind. Außerdem sind sexuclle Interaktionen kein notwendiger Bestandteil intimer Berichungen, weil Intimität Sexualität nicht zwangsläufig cinschließt. Kombiniert man die 1)efinition mit den notwendigen Bestimmungen von Intimität als solcher (vgl. Prager u. Roberts 2004, 46-47), ergeben sich drei wesentliche Elemente von intimen Bezichungen:

1. Intimität: Fine Bezichung, in der man sich nicht in irgendeiner Weise geistig, emotional oder körperlich berührt und begegnet, wäre keine intime Beziehung, sondern ein kontaktloses Nebeneinander. Damit eine intime Verbindung zwischen beiden l'artnem cntstehen kann, muss nach dem intimacy process model (Reis u. Shaver 1988) ein Partner dem anderen gegenüber verbal oder nonverbal persönlich relevante Information, Gedanken oder Gefühle enthüllen, wobei das nonverbale Mitteilen von (icfühlen am wichtigsten für das Empfinden von Intimität ist (vgl. Laurenceau u. Kleinman 2006). Das (icgenüber muss im Gegenzug für diese persönliche Mitteilung empfänglich sein und sein Verständnis und Interesse dafür deutlich machen, so dass sich der Partner, der die Botschaft sendet, verstanden fühlt. Besonders tiefe Formen der Selbstoffenbarung betreffen verletzliche Gefühle, mit denen man sich dem Partner gegenüber schutzlos zeigt. Dic mitgeteilten Gefühle müssen dabei nicht ausschließlich positiv, sondern können auch negativ sein wie zum Beispiel Reue oder Traurigkeit.

2. Vertrautheit: Damit sich aus den intimen Begegnungen eine intime Bezichung cntwickelt, müssen diese Begegnungen so aufeinander aufbauen, dass sich daraus cin Bewusstsein der gencinsamen Geschichte, 
wechselseitiges Wissen um das Innenleben des anderen und Verständ nis für ihn ergibt, das über die intimen Begegnungen hinausreicht. Eine Bezichung, in der es keinen andauemden liufluss aufeinander gäbe, sondern in der man sich jeden 'Tag aufs Neue kennenlemen müsste, würde nicht als intime Bezichung betrachtet. Ietztlich ist das entscheidende Abgrenzungsmerkmal von intimen zu anderen persönlichen Bezichungen, dass sich zwei Menschen auf persönliche Weise kennen. Un sich entwickeln zu können, muss dieses intime Wissen um den anderen und die Bezichung zu ihm kognitiv repräsenticrt sein. Eine Voraussetzung, die im Alltag selbstverständlich ist, aber infolge einer Demenz fragwürdig werden kann.

3. Positivität: Damit die intime Bexichung auf Dauer bestehen kann, ist ein positives Eingehen auf den anderen notwendig. (rrundsätrlich geht es darum, dass man dem anderen scine Aufmerksamkeit widmet und verbal oder nonverbal seine positiven Gefühle deutlich macht. Dic Bedeutung des positiven Umgangs miteinander wird deshalb hervorgehoben, weil man im Alltag dazu neige, die positiven Aspekte fü selbstverständlich zu nehmen, während sich die negativen als Störungen in den Vordergrund drängen (vgl. Jellouschek 2011). Erklärt wir diese selektive Wahrnehmung mit adaptiver Signifikanz, der I läufigkeit und Salienz, des negativen Verhaltens und seiner eindeutigeren Interpretation: Unangenehme, seltenere Interaktionen stechen mchr hervor und werden eindeutiger interpretiert als positive. Außerdem würden Menschen generell potentiellen Bedrohungen mehr Aufmerksamkeit schenken als freudigen Ereignissen (vgl. Blieszner 2006).

Diese drei Elemente bilden die Voraussetzung damit man überhaupt von einer intimen Beziehung sprechen kann. Das Ausmaß und dic Qualität der intimen Begegnung hängt davon ab, wic tief die Selbstoffenbarung ist, wie stark man positiv engagicrt ist und wie ausgeprägt und zutreffend das wechselseitige Wissen und Verstehen ist (vgl, l'rager u. Roberts 20(14).

\section{Anforderungen zur Aufrechterhaltung von Beziehungsfähigkeit}

Aus den obigen drei Elementen lassen sich die Anforderungen ableiten, die Partner erfüllen müssen, wenn sie intime Begegnungen trotz kognitiver, emotionaler und motivationaler Einschränkungen erhalten wollen:
1. Intimität: Dic Erfahrung von Intimität setzt verbale oder non-verbale Kommunikation voraus, die die eigenen Gefühle ausdrücken kann, ebenso wie die Wahmehmung der Gefühle des anderen und die cmpathische Reaktion darauf.

2. Vertrautheit: Da Intimität zwar nur in Augenblicken empfunden wird, eine intime Bezichung aber aus dem erworbenen Wissen und Verstehen dem anderen gegenüber besteht, muss es irgendeine Form der Erinnerung an den Partner und die Beziehung zu him geben. Diese Erinnerung bezieht sich nicht nur auf verbal Mitgeteiltes, sondern auch auf andere sinnliche Erfahrungen wie Geriiche, die Stimme des Partners oder gemeinsame Rituale, die zur Identität als Paar beitragen können. Das Gefühl der Vertrautheit setzt aber nicht nur das Wissen um das Innenleben des anderen voraus, sondern auch, dass dieses Wissen nicht im Wicierspruch zum eigenen Erleben des Partners steht. Dafür müssen sich die Partner authentisch dem anderen gegenüber öfnen. Hilfreich ist dafür cin kongruentes Selbst, das eigene Gefühle und Erfahrungen nicht deshalb abwchrt, weil sie dem eigenen Selbstkonzept widersprechen (Prager u. Roberts 2004).

3. Positivität: Wenn die intime Beziehung erhalten werden soll, ist es nicht nur notwendig, eine gewisse Wechselscitigkeit an intimen Begegnungen zu haben, sondern auch, dass man grundsätzlich achtsam mit clem Partner umgcht und scine Wertschätzung ihm gegenüber deutlich macht. Intimität kann zwar auch durch negative oder gar feindselige Gefühle entstehen. Ohne positive Interaktionen wird aber die Enthüllung der eigenen Verletzlichkeit nicht möglich sein, weil man dom Partner vertrauen können muss, dass er das Wissen um die eigene Vorletzlichkeit nicht gegen einen verwendet.

\section{Beziehungsrelevante Fähigkeiten von Menschen mit Demenz, die erhalten bleiben können}

Welche Fähigkeiten bleiben crhalten, die Menschen mit Demenz und ihre Partner nutzen könncn, um eine intime Vorbindung zu erhalten? Ich gebe im lolgenden einen Überblick über einige empirische Befunde 7.4 den verbleibenden Fähigkeiten von Menschen mit Demenz, die verdeutlichen, inwieweit Menschen mit Demenz und ihre Partner die drei obigen Voraussetzungen in reduzierter form erfüllen können. Da es keine systematischen Überblicksarbeiten zu den verbleibenden Häluigkeiten von Menschen mit 
Demenz über alle Demenzen hinweg gibt, beziehen sich diese Studien meist auf den bekanntesten Typ, die Alzheimer-1)emen\%. Finschränkend muss hervorgehoben werden, dass die Veränderungen durch die Demenz moist aus der Sicht der Partner erhoben werden.

Zwar gibt es bestimmte Fähigkeiten, die als Voraussetzungen für intime Beziehungen gelten können (s.o.), aber Beziehungsfähigkeit ist meines brachtens nur als das Ergebnis der Anstrengung beider Partnet sinnvoll zu begreifen. Das gilt gerade da, wo der Mensch mit Demenz weiterhin wichtige Fähigkeiten für intime Bezichungen mitbringt, die aber zunchmend vom Partner aktiv genutzt werden müssen (s. u.). Ich spreche daher hier von liähigkeiten, die zum Aufrechterhalten von Beziehungen relevant sind.

\section{Intimität}

Durch die kognitiven Einschränkungen und Veränderungen im Erleben und Verhalten schwindet zumehmend dic Möglichkeit, dass die Partner eine Verbindung zum Menschen mit Demenz herstellen können. Das betrifft zunächst die geistige Nähe, weil der Austausch über intellektuelle Themen durch Gedächtnisstörungen und Einschränkungen der Denkfähigkeit und Kommunikationsfähigkeit erschwert wird. bbenso kann bei fortgeschrittener Demenz auch die körperliche Nähe abnehmen, weil zum Beispicl auch Sexualität von beiden Partnem nicht mehr gewollt wird: Beim Partner kann sich das Begehren ändern und beim Menschen mit Demenz die Apathic verstärken (vgl. Derouesné 2005).

Lange erhalten bleiben kann allerdings eine emotionale Nähe. Zwar kann chon bei Beginn der Demenz die verbale Kommunikationsfähigkeit durch Wortfindungsstörungen, Schwierigkeiten bei der geteilten Aufmerksamkcit durch kureitigen Behaltens eingeschränkt seir. Menschen mit Demenz verfügen aber auch im snäten Stadium über die Fähigkeit, nonverbale Mitteilungen fer Beziehungsehene au machen und zu verstehen. Hier wird Menschen mit Demenz. sogar cine größere Sensibilität als Menschen olune Demenz auf den nonverbalen Kommunikationskanälen zugesprochen, was sic empfänglicher machen soll für emotional ausgedrückte Beziehungsbotschaften (vg). Kümmel u. Haberstroh 2010; Sachweh 2008). In der Regel verschlechtert sich allerdings bei Menschen mit Alzheimer-Demenz dic Wahrnehmung von Fmotionen über Mimik und (iestik vom leichten bis zum mittleren Stadium (vgl. Blessing et al. 2014). Belastend ist ebenfalls ihre Apathie, die die bäu- figste Veränderung im Firleben ist (vgl. Schäufele et al. 2006). Hier kann der Partner besonders starke Trauer über den Verlust an Intimität und Wechselscitigkeit empfinden.

Paradoxerweise kann in einigen Fällen der Mensch mit Demenz, durch die Finschränkungen der kognitiven Kontrolle des eigenen Verhaltens in manchen Fällen als authentischer als vor Beginn der Demenz wahrgenommen werden, weil er frühere Hemmungen und die lassadenhaftigkeit des Alltags ablegt und dadurch offener wird (vgl. Kitwood 1995). Umgekehrt sind abstrus und unverständlich wirkende Verhaltensweisen des Menschen mit Demenz besonders belastend, weil sie ein Gefühl der Entfremdung auslösen (s.o.).

lixperimentelle Studien zur Theory of mind (der Fähigkeit, anderen und sich selbst psychische Zustände zuzuschreiben) bei Alzheimer-Demenz und der Verhaltensvariante der fronto-temporalen Demenz zeigen, dass bei beiden Demenzen, aber besonders deutlich bei der fronto-temporalen Demenz, mit der Krankheitsdauer und der allgemeinen kognitiven Einschränkung Defizite bei der Perspektivenübernahme zunehmen (vgl. Bora et al. 2015). Gerade diese Aspekte der sozialen Kognition sind besonders entscheidend fïr wechselseitig befriedigende Beziehungen, weil man ohne sie nicht sensibel auf den anderen eingehen kann. Allerdings gibt es auch Hinweise dafür, dass zumindest in Einzelfällen Menschen mit Demenz mindestens bis ins mittlere Stadium hincin Empathie zeigen, sich beispielsweise in clie psychische Belastung des Pflegenden einfühlen, ihn deshalb cmotional unterstützen und die Wechselseitigkeit erhalten können (vgl. Ablitt et al. 2010).

\section{Vertrautheit}

Wie oben ausgeführt, ist das Ciedächtnis das verbindende Gewebe in intimen Bezichungen. Diese Verbindung wird durch Demenz gefährdet: (ierade bei der Alzheimer-Demenz ist das episodische Gedächtnis für neue Frlebnisse früh becinträchtigt, weiter zurückliegende Erlebnisse können dagegen noch erimnert werden. Das semantische Gedächtnis ist etwas robuster, weil Weltwissen überlernt ist, aber auch das wird schlechter. Bei schwerer Demenr. ist dann auch das autobiographische Langzeitgedächtnis beeinträchtigt.

Mit dem Fortschreiten der Demenz nimmt also nicht nur die Möglichkeit ab, über neue Erlebnisse die Verbindung zu stärken, sondern auch die gemeinsame Erinnerung an dic Spuren, die man im Icben hinterlassen hat 
(wie $x$. B. den Hausbau usw.). Der l'artner wird hier zunchmend zu einer Art Hilfsgedächtnis für den Menschen mit Demenz, um so die Identität als Paar für beide zu bewahren. Vom eigenen Partner aufgrund schwerer De menz nicht mchr erkannt zu werden, ist deshalb zumeist mit dem Gefühl eines riesigen Verlustes und entsprechender Trauer verbunden (vgl. Zank u. Schacke 2007).

Auch wenn hier große Verluste von Vertrautheit und Bewusstscin der gemeinsamen Geschichte zu beklagen sind, sollten die erhaltenen Elemente des impliziten Gedächtnisses nicht übersehen werden, die es ermöglichen, cinen Teil der Vertrautheit zu erhalten. Zu den verbleibenden emotionalen Fähigkeiten von Menschen mit Demenz gehört das implizite emotionale Lernen. Dic Ciefühle, die Menschen mit Demenz in der Begegnung nit anderen Menschen erleben, können auch dann fortbestehen und durch neue Erfahrungen verändert werden, wenn diese lirlebnisse nicht bewusst erinnert werden: So gibt es experimentelle Nachweise, dass das emotionale Lernen, bei dem Emotionen gegenüber Personen durch positive oder negative Informationen verändert werden, bis mindestens ins mittlere Stadium der Alzheimer-1)emenz erhalten bleibt (vgl. Blessing et al. 2014). Auch wenn im Experiment die Dissoziation von bewusster Erinnerung und implizitem emotionalen Icrnen nur für die Dauer eines Tags nachgewiesen ist, zeigt dieser Befund, dass Menschen mit Demenz trotz des Verlustes des episodischen Gedächtnisses - zumindest für einen bestimmten Zeitraum - auf der Basis einer fortdauernden emotionalen Bindung zu anderen Personen nachhaltige Bezichungserfahrungen machen können. 1)as erhalten gebliebene implizite (jedächtnis ermöglicht, dass selbst bei Verlust der bewussten Erinnerung neue Beziehungen eingegangen werden und alte fortbestehen können.

Für das (iefühl, dass man den anderen persönlich kennt, ist es ebenso wichtig, dass das Wissen um den Partner ihm auch zu cotsprechen schein (s.o.). Entsprechend belastend ist das Gefühl, den Menschen mit Demenz. gar nicht mehr zu kennen, weil er völlig anderes als früher zu reagieren scheint. Das Personensein des Menschen mit Demenz, das heißt die Kontinuität seincr Person, ist aber nicht ausschließlich von intakter Kognition, also dem expliziten autobiographischen Gedächtnis abhängig. Aus leibphänomenologischer Sicht wird das Selbst als verkörpertes Selbst verstanden. Da wir nur leiblich in den Austausch mit anderen und der Welt treten können, können Teile des Selbst, unsere leiblich gemachten Erfahrungen und Gewohnheiten (\%.B. unser Habitus), so in den Körper sedimentieren, das sie Teil des impliziten "L.eibgedächtnisses " werden (vgl. luchs 2010). liin
Teil der Persönlichkeit des Menschen mit Demenz kann deshalb auch für andere wahrnehmbar erhalten bleiben, weil es sich dabei zum Beispiel um (iewohnheiten handelt, wie das Auftreten, die Gebärden, den Gang oder die Stimme, die unabhängig von bewusster Reflexion sind.

\section{Positivität}

Schwindende geistige, körperliche und emotionale Nähe, herausfordernde Verhaltensweisen sowic Apathie und Entfremdungsgefühle können dazu führen, dass Partner nur wenig positive Rückmeldung und Aufmerksamkeit vom Menschen mit Demenz bekommen. Entsprechend wird Demenz allgemein ein negativer finfluss auf die Bezichungsqualität zugesprochen, weil Wechselseitigkeit, offene Kommunikation und gemeinsame Er tebnisse reduriert sind.

Allerdings wird in Übersichtsarbeiten auch betont, class positive $\Lambda$ spekte wie Liebe, Wärme und Nähe erhalten bleiben können (vgl. Ablitt et al 2009). Auch Menschen mit fortgeschrittener Demenz können durchaus ihre Dankbarkeit und Wertschätzung ihrem Partner gegenüber ausdrücken. Entscheidend ist nicht, dass jeder den gleichen Beitrag leistet, sondern dass man überhaupt das Ciefühl hat, erkannt und wertgeschätzt zu werden.

Tabelle 1 gibt nochmals einen Überblick sowohl über die Fähigkeiten, die für intime Beziehung nötig sind, als auch über die Fähigkeiten von Menschen mit Demenz, die für die Aufrechterhaltung intimer Beziehungen bis zum mittleren Stadium der Demenz, erhalten bleiben können.

Tabelle 1: Bis zum mittleren Stadium erhaltene Fähigkeiten für intime Be ziebungen von Menschen mit Demenz

\begin{tabular}{|c|c|c|}
\hline $\begin{array}{l}\text { Elemente intimer Bezic- } \\
\text { hungen }\end{array}$ & $\begin{array}{l}\text { Not wendige Fähigkeiten } \\
\text { für intime Bezichungen }\end{array}$ & Erhaltcne Fähigkeiten \\
\hline \multirow[t]{4}{*}{ Intimität } & Kommunikation & Nonverbale Konmunikation \\
\hline & Enotionserkcmung & $\begin{array}{l}\text { an auditiven Mcrkmalen (Stimm- } \\
\text { klang, Musik, Gerä̈tsche) }\end{array}$ \\
\hline & $\begin{array}{l}\text { Emotionserteben und Emo- } \\
\text { tionasansdruck }\end{array}$ & durch Mimik \\
\hline & Eimpathic & für die Belastung des Partners \\
\hline
\end{tabular}




\begin{tabular}{|c|c|c|}
\hline $\begin{array}{l}\text { Elemente intimer Bezie- } \\
\text { lungen }\end{array}$ & $\begin{array}{l}\text { Notwendige Fähigkeiten } \\
\text { für intime Bezichungen }\end{array}$ & Erhaltene Fähigkeiten \\
\hline \multirow[t]{2}{*}{ Vertrautheit } & $\begin{array}{l}\text { Reprïsentanz des anderen } \\
\text { und der Beziehung zu ilum }\end{array}$ & $\begin{array}{l}\text { durch emotionales Gedächnis und } \\
\text { Lernen }\end{array}$ \\
\hline & Kontinuität der l'erson & durch Leibged lichunis, Authentizität \\
\hline \multirow[t]{3}{*}{ Positivität } & Positive Interaktionen & Erhalt der Licbe \\
\hline & & Wertschätzung \\
\hline & & Bindung \\
\hline
\end{tabular}

\section{Schlussfolgerung:}

\section{Als Paar leben und lieben mit uneindeutigem Verlust}

Menschen mit Demenz bringen durchaus Fähigkeiten mit, die es ihnen er möglichen, intime Bexiehungen zu anderen Menschen einzugchen (siehe Tab. 1): Auch wenn dic Nähe auf geistiger und körperlicher Ebone schwindet, so kann doch eine emotionale Nähe bestehen bleiben. Zur bleibenden Verbindung trägt auch bei, dass Menschen mit Demenz, nachhaltige emotionale Erfahrungen machen können und ihr. Wesen präscnt bleiben kann. Die Verluste in der Bezichung zum Menschen mit Demenz, und die Trauer darüber gehören für die Partner zwar zu den größten Belastungen (vgl. Zank u. Schacke 2007). Dennoch können positive Aspekte verbleiben, wenn l'artner Zuncigung füreinander empfinden und eine starke Bindung ancinander haben (vgl. Prager u. Roberts 2004).

Der Zwiespalt zwischen dem Verhust an geistiger und körperlicher Nähe bei potentiell erhaltener emotionaler Nähe lässt sich mit Pauline Boss als "uneindeutiger Verlust " des Partners begreifen. Dieser Verlust sei deshalb so schwer zu ertragen, weil er zu chronischer und blockierter Trauer fülure und keinen Abschluss crmögliche. Boss (2011) plädiert für cin flexibles Sowohl-als-auch-Denken, das sowohl die Verluste als auch das Erhaltene in der Bezichung zum Mcnschen mit Demenz anerkennt und durch eine stärkere Bindung an andere Menschen aushaltbar macht.

Welche Kompetenzen können nun beide Partner nutzen, um trotz des uneindeutigen Verlustes ihre intime Bezichung möglichst lange zu erhalten? Der Partner kann seine Fähigkeit zur einfühlsamen Kommunikation nutzen, d.h. dem Menschen mit Demenz, in seine Innenwelt folgen, die Konfrontation mit Defiziten vermeiden, sich auf seinen Standpunkt einstellen, was der Mensch mit Demenz scinerseits nicht mehr leisten kamn. Damit wird der Partner zu demienigen, der die Beziehung aktiv durch viel Kreativität und Flexibilität gestaltet. Der Beitrag des Menschen mit Demenz verschiebt sich hier vom Tun zum Sein, was seinen Beitrag aber nicht weniger wichtig macht: Das Ausdrücken und Verstehen von nonverbalen Bezichungsbotschaften, das erhalten gebliebene "Wesen « des Menschen mit Demenz und Ausdrücken von Dankbarkeit oder die Akzeptanz, von Freiräumen können es Menschen mit Demenz und ihren Partnern ermöglichen, eine emotionale Nähe zu erhalten.

Bei fortgeschrittener Demenz, ruht die Beziehung deshalb zum einen auf der erhaltenen emotionalen Nähe zwischen beiden Partnern und zum anderen auf dem Versuch des Partners, den Menschen mit Demenz wegen seines Verständnisses für die emotionale Bezichungsbotschaft möglichst partnerschaftlich in lintscheidungen mit einzubeziehen (vgl. Bödecker 2015).

\section{Danksagung}

Ich danke dem Netzwerk Alternsforschung in Heidelberg für die finanziclle Unterstützung beim Verfassen dieses Beitrags.

\section{Literatur}

Ablitt $A$, Jones $G$, Muers J (2010) Awareness of carer distress in people with dementia. International Jourmal of Geriatric Psychiatry 25(12): 1246-12.52

Ablitt $\Lambda$, Jones $G$ V; Muers J (2009) Living with dementia: $\Lambda$ systematic revicw of the influence of relationship factors. $\Lambda$ ging \& Mcntal I lealth 13(4): 497-511.

Blessing $\Lambda$, Forstncier $\$$, Eschen $\Lambda$ (2014) Emotionen als Wirkfaktoren psychosozialer Interventionen bei Alzheincr-Demenz. Zeitschrift für I'sychiatrie, Psychologie und I'sychotherapic 62(3): 191-199.

Bödecker I: (2015) Paarkonflikte bei Demenz: Von Finden einer neuen Balance zum Fïnden einer neuen Basis. Weinheim (Beltz-Juventa).

Blieszner R (2006) Close Relationships in Middle and Late $\Lambda$ dulthood. In: Vangelisti $\Lambda \mathrm{L}$ I'erlman D (Itg) (2006) The Cambridge handbook of personal relationships. New York (Cambridge University Press) 211-227

Bora l:, Walterfang $\mathrm{M}$, Velakoulis $\mathrm{D}$ (2015) Theory of mind in behavioural-variant frontotemporal dementia and Alzheimer's disease: a meta-analysis. J Ncurol Neurosurg l'sychiatry 86 $714-719$

Boss IG (2011) Loving Someone Who I Ias Denentia: How to Find I lope While Coping with Stress and Gricf. San lirancisco (John Wiley \& Sons).

Bradbury 'IN, Kancy BR (2010) Intinnate relationships. New York (W. W. Norton \& Company). 
Danschen (i, Schönecker D) (2013) Selbst philosophieren. Kïn Methodenbuch. Berlin (I) (iruyter).

Davies S, Nolan M (2008) Attending to relationships in dementia care. In: Downs M, Bowers B (1/g) Excellence in dementia care: Research into practice. Maidenhend (Open University P'ress) 438-454.

Derouesné C (2005) Scxualité et démences. Psychologie \& Neurol'sychiatric du Vicillissement $3(4): 281-289$.

Fuchs $T$ (2010) Das Leibgedächtnis in cler Demenz, In: Kruse $\Lambda$ (I lg) Lebensqualität bei Demenz? Zum gesellschaftlichen und individuellen Ungang mit einer Grenzsituation im $\Lambda$ trer. Heidelberg ( $\Lambda$ kadenische Verlagsgesellschaft $\Lambda K \Lambda$ ) 231-242.

Hansson RO, Daleiden liL, Hayslip B Jr. (2004) Relational Competence across the Life Span. In: I ang IR, Fingerman KL ( $\mathrm{Ig})$ Growing together: Personal relationships across the life span. Cambridge (Cambridge University l'ress) 317-340.

Jellouschek H (2011) Wonn Paare älter werden. Die Liebe nen entdecken. Preiburg, im Breisgau (Herder).

Kitwood 'I (1995) Positive long-term changes in dementia: Some prelininary observations. Journal of Mental I lealth 4(2): 133-144.

Kümmel $\Lambda$, Haberstroh J (2010) Kommunikationsfähigkeit demenzkranker Menschen. In: Haberstroh J, Pantel J (I Hg) Demenz psychosozial behandelin. P'sychosoziale Interventionen bei Demenz in Praxis und Forschung. Heidelberg ( $\Lambda$ kademische Verlagsgesellschaft $\Lambda K \Lambda$ ) $269-280$.

Laurenceau J, Kleimman BM (2006) Intimacy in l'ersonal Relationships. In: Vangelisti $\Lambda$ L, Perlman D) (l. Ig) The Cambridge handbook of persomal relationships. New York (Cambridge University Press) 637-653.

Mackae 1] (2011) Self and other: 'The importance of social interaction and social relationships in shaping the experience of early-stagc $\Lambda$ Izhcimer's disease. Journal of $\Lambda$ ging Studlies 2.S(4): 445-456.

Prager KJ, Roberts L.J (2004) Deep Intinate Connection: Self and intimacy in Couple Relationships. In: $\Lambda$ ron $\Lambda$, Mashek D) (Hg) Handbook of closeness and intimacy. Mahwah (lawrence Erlbaum $\Lambda$ ssociates) 43-60.

Reis HI, Shaver P' (1988) Intinacy as an interpersonal process. In: Duck S, I Iay DI', I Iobfoll SI:, Ickes W, Montgoncry BM (I / $\mathrm{g}$ ) Handbook of personal relationships: 'lheory, research and interventions. Oxford (John Wiley \& Sons) 367-389.

Sachweh S (2008) Spurenlesen im Sprachdschungel: Kommunikation und Verständigung mi demenzkranken Menschen. Bern (Verlag Hans Huber).

Schäufele M, Köhler L, leufel S, Weyerer S (2006) Betreunng von demenziell erkrankton Menschen in Privathaushalten: P'otenziale und Grenzen. In: lingels D), Schncekloth U (I Ig) Selbständigkeit und Hilfebedarf bei älteren Menschen in Privathaushalten: Pflcgearrangcments, Demenz, Versorgungsangebote. Stuttgart (Kohlhammer) 103-14.5.

Stechl l? (2006) Subjektive Wahruehmung und Bewältigung der Denenz im lirühstacliuns: line qualinative Intervicwstudie mit Betroffenen und ihren Angehörigen. lserlin (Köster).

Zank S, Schacke C (2007) Projekt Längsschnittstudie zur Belastung pllegender Augehr̈rige von demenziell lirk rankten (LI:ANDLER): Abschlussbericht l'hase 2: Längsschnittergebnisse der LIEANDER Studic.
Korrespondenzadresse:

1)r. phil. Hlorian Bödecker

Zentrum für allgemeine wissenschaftliche Weiterbildung

Universität Ulm

Albert-Finstcin-Allec 11

$89081 \mathrm{Ulm}$

F,-Mail: florian.boedecker@uni-ulm.de

\section{Zum Autor}

Florian B̈̈decker, Jg. 1975, Dr. phil. Dipl.-Päd., Wissenschaftlicher Mitarbeiter am ZAWiW der Universität Ulm. Interessengebietc: Paarkonflikte bei Dement, Forschungsethik und Forschungsmethodik bei kognitiver Beeinträchtigung, psychosoziale Beratung in Alter, Geragogik, Alters- und Krankheitstheorien, Gesundheits- und Pflegesystem im Kapitalismus und Entwicklung innovativer Bildungsangebote für Ẍltere. 